\title{
ON THE PRIME EXAMPLE OF PROGRAMMING
}

\author{
Paul Pritchard \\ Department of Computer Science \\ University of Queensland \\ ABSTRACT
}

\begin{abstract}
The problem of finding prime numbers has been much favoured by expositors of modern programming methodology. In this paper the problem is tackled once more, but from a broader view of the programing process than has previously been taken. The initial steps toward a solution parallel those in the well-known presentations of Dijkstra and Wirth. However a general program transformation is shown to capture the clever "inventive step" in Dijkstra's solution, and another is employed to lead naturally to an alternative program. Complexity analyses of the two programs show that the latter is significantly more efficient.
\end{abstract}

\section{INTRODUCTION}

The problem of finding prime numbers enjoys a special status as an exemplary exercise for modern programming methods. Dijkstra, chooses it as his "first example of step-wise program composition" in what is probably his most widely read essay, "Notes on structured Programming"; Wirth (1973) develops a variant of Dijkstra's program for the problem as a key example in his "Systematic Programming"; Hoare (1972) employs a given abstract algorithm for the problem to illustrate the transition from abstract data structures to their realizations in a programming language.

We choose herein to tackle the problem once more, with a view to exemplifying the total programing process. For our purposes we take this to encompass the following stages:

1. problem specification;

2. design of an initial solution;

3. creation of alternative solution(s);

4. evaluation and comparison of the solutions.

The numbering of these stages is not meant to suggest a rigid chronological order; considerable interaction would be expected in the later stages.

As with Dijkstra and Wirth, program correctness is the dominant concern in all but the fourth stage. In this respect, we show that the depenäence on a "deep" theorem of number theory of Dijkstra's (and wirth's) program can easily be removed; we also bring to light a subtle verification condition for Wirth's program. This condition is a complicated number-theoretic assertion whose validity is unclear.

1. Dijkstra (1972), p.26. 
We hope to show that program transformations have an important role to play in both the creation of an initial solution and subsequent solutions. We show that a general optimising transformation gives a much better account of the "inventive step" in Dijkstra's solution. Furthermore, another general transformation is the crucial step in the development of an alternative program. our final concern is with analysing the time and space complexity of the two solutions; we reference results which clearly demonstrate the superior efficiency of the second program.

\section{THE PROBLEM, AND THE DEVELOPMENT OF AN INITIAL SOLUTION}

We choose the following formulation of the problem:

find all primes not greater than a given integer $N>1$.

Dijkstra and Wirth actually find the first $n$ primes, but this is not an important difference (Dijkstra is uncharacteristically concrete with $n=1000$ ).

Our initial design steps parallel those of Dijkstra and wirth; $p_{i}$ is used to denote the $i$ 'th prime.

Step 1: accept 2 as the first prime, then examine successive odd integers $3,5,7 \ldots$ for primeness, stopping when the next candidate exceeds $\mathrm{N}$.

Step 2: (refining the test for primeness): since an odd number $\mathrm{x}$ is prime if and only if it has no odd prime factors other than itself, we decide to divide $\mathrm{x}$ by $\mathrm{p}, \mathrm{p}, \ldots$ successively until a factor is found or no candidate factors remain. So it is decided to store $p_{i}$ in an array (as $p[i]$ ) when found, in order that primes be available for the primality test.

Step 3: (deciaing when to stop testing): if $x$ is composite, its smallest (prime) factor must be $\leq \sqrt{x}$. So we must stop testing if

(i) a factor is found, or

(ii) we run out of primes $<x$, or

(iii) the next prime $p$ to be tried satisfies $p^{2}>x$.

Hence, if $k$ primes have been found thus far, the upper bound $1 \mathrm{im}$ on the index of primes to be tried is given by

$$
\lim =\left\{\begin{aligned}
k+1, & \text { if } p[k]^{2} \leq x, \\
1+1, & \text { where } 1 \text { is the smallest index such that } \\
& x<p[1+1]^{2}, \text { otherwise. }
\end{aligned}\right.
$$


It is incumbent on the programmer to ensure that (1) is invariantly true before doing the primality test on $x$. Since Iim is a function of $x$ only, we seek to adjust its value for each new value of $x$. So suppose (1) holds with $x, k$ and lim equal to $\mathrm{xl}, \mathrm{kl}$ and $\operatorname{liml}$ respectively, just before testing $\mathrm{xl}$. Let $\mathrm{x} 2$ and $\mathrm{k} 2$ be the new values of $\mathrm{x}$ and $\mathrm{k}$ after completing the test and incrementing $x$. Suppose liml $>\mathrm{k} 2$. This can only happen if $\lim 1=k l+1$ and $k 2=k l$ (because $x l$ was composite). So by (1), $p[k 2]^{2} \leq \mathrm{x} 2=\mathrm{xl}+2$, so 1 im should not be changed. The remaining case is $\operatorname{liml} \leq \mathrm{k} 2$. Here lim must be increased if $\mathrm{p}[\operatorname{liml}]^{2} \leq \mathrm{x} 2$, and it can only increase by 1 since $\mathrm{k}$ and 1 (in(1)) can increase by at most 1. The resulting program is given in Figure 1

const $\mathrm{N}=$ ?; pbound $=$ ?;

$\overrightarrow{\operatorname{var}} \mathrm{p}:$ array $[1$. pbound] of integer;

$i, x, k, 1 i m$ : integer;

prim: Boolean;

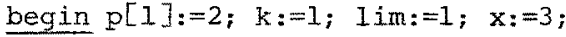

while $x \leq N$ do

begin if $1 \mathrm{im} \leq \mathrm{k}$

then if $p[1 \mathrm{im}]^{2} \leq \mathrm{x}$ then $1 \mathrm{im}:=1 \mathrm{im}+\mathrm{I}$;

i. $:=2$; prim: = true;

while prim and $(i<I i m)$ do

begin prim: $=\langle x$ is not divisible by $p[i]>$;

$i:=1+1$

end;

if prim

then begin $\mathrm{k}:=\mathrm{k}+1$;

$\mathrm{p}[\mathrm{k}]:=\mathrm{x}$

end;

$\mathrm{x}:=\mathrm{x}+2$

end.

end

ETGURE 1 .

The Dijkstra and wirth programs differ from our program only in omitting the test $1 \mathrm{im} \leq \mathrm{k}$. Since $\mathrm{p}[\mathrm{i} \mathrm{im}]$ is not defined for $1 \mathrm{im}>\mathrm{k}$, the correctness of their programs depends on showing that $1 \mathrm{im} \leq \mathrm{k}$ is invariantly true. They do this by appealing to what knuth calls "a deep result of number theory, namely .... we always have

$$
p_{n+1}<p_{n}^{2 n} \cdot
$$

2. D. Knuth, quoted in Dijkstra (1972), p.34. 
This is unfortunate in a pedagogical example chosen because "its mathematical background is so simple and familiar". " we have shown above that the programmer who is unaware of this fact is not disadvantaged (at this stage), as it is only of very minor importance.

We now proceed to step 4 (refining $<x$ is not divisible by $p[i]>$ ): the obvious choice here is

$$
(x \text { div } p[i]) * p[i] \neq x \text {. }
$$

Dijkstra and wirth surpxisingly (to the reader) do not stop at this point, and give rather strange reasons for continuing the process of refinement - Dijkstra: "to give the algorithm an unexpected turn we shall assume the absence of a convenient remainder computation" ${ }^{14}$; Wirth: "Let us, however, assume that the program is to be developed without the availability of an explicit division operator" 5 . We feel these invitations are unconvincing; what is hardware division for if not to divide?

But it is not difficult to motivate the move. It is in fact an example of a general optimizing transformation we might express as follows :

$$
\begin{aligned}
& \text { if, in a loop, } x \text { is incremented by } \delta(x) \text { before calculating } \\
& f(x) \text {, and } f(x+\delta(x))=g(f(x), \delta(x)) \text { where } g(c, y) \text { is } \\
& \text { easily calculated for small values of } y \text {, then save the } \\
& \text { (previous) value of } f(x) \text { in } f x \text { and compute with } g(f x, \delta(x)) \text {. }
\end{aligned}
$$

(Dijkstra actually addresses this same phenomenon later in his essay.) Furthermore, a heal thy respect for the cost of division and multiplication is sufficient stimulus to look for this situation in our example.

$$
\begin{aligned}
& \text { In our program, given } f x=(x \text { div } p[i]) * p[i], \\
& (x+2 \text { div } p[i])^{*} p[i]=\left\{\begin{array}{l}
f x+p[i], \text { if } f x+p[i] \leq x, \\
f x, \text { otherwise. }
\end{array}\right.
\end{aligned}
$$

Since the above is clearly easy to compute, we decide to introduce an array of multiples $\mathrm{m}[i]$ of $\mathrm{p}[\mathrm{i}]$ for $i<1 \mathrm{im}$. This amounts to keeping

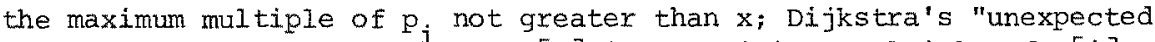
turn" is a slight variation - $\mathrm{m}[\mathrm{i}]$ is the minimum multiple of $\mathrm{p}$ [i] not less than $x$. The natural place to adjust $m[i]$ is in the loop for the primality test, which wirth codes as follows:

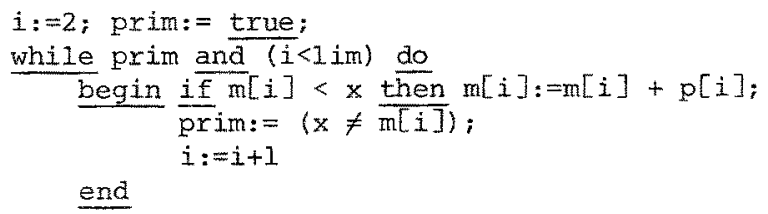

3. Dijkstra, op.cit., p. 27.

4. Ibid., p.37.

5. Wirth (1973), p.140, 
But there is a trap here! If $\mathrm{m}[\mathrm{i}]$ is only calculated when needed, we must be sure it cannot lag too far behind $x$. Dijkstra solves this with

$$
\text { while } m[i]<x \text { do } m[i]:=m[i]+p[i] \text {, }
$$

which is guaranteed to preserve the appropriate invariant. Wirth's program has an implicit verification condition $\mathrm{VC}$, on whose validity the correctness of his program stands or falls.

$$
\begin{aligned}
& \text { VC: In any interval } n \cdot p_{i}<x<m \cdot p_{i}, n \geq p_{i}, n \text { and } m \text { coprime } \\
& \text { to all } p_{k} \text { with } k<i \text {, there are distinct integers } x_{j} \text {, } \\
& 1 \leq j \leq m-n-1 \text {, such that for each } j, x_{j} \text { is coprime to ail } \\
& p_{k} \text { with } k<i \text { and } x_{j}>(n+j-1) \cdot p_{i} \cdot \quad \text { (Pritchard, 1979a) }
\end{aligned}
$$

We know of no proof of VC, and expect a proof would be quite complicated. Note that if $\mathrm{VC}$ is not valid, it is still possible that wirth's program finds the expected prime numbers. A counterexample to $\mathrm{VC}$ demonstrates the incorrectness of the asserted program, which means that correct input/output behaviour of "the program would essentially be fortuitous and undemonstrated.

\section{THE DEVELOPMENT OF AN ALTERNATIVE PROGRAM}

The purpose of the inner loop in our first solution/program is to check if $x$ is equal to (at least) one of a number of values $m[i]$, which, when computed, satisfy

$$
\mathrm{m}[\mathrm{i}]=\text { the least multiple of } \mathrm{p}[\mathrm{i}] \geq \mathrm{x} \text {. }
$$

Now at an abstract level there is an obvious transformation applicable here, viz. to regard the primality test as a test for membership of $x$ in the set $M=\{m[i] \mid 1 \leq i<1 \mathrm{im}\}$. Furthermore, it is easy to specify the value of $M$ for the next value of $x-i t$ is just

$$
M-\{x\} \cup\{x+p[i] \mid m[i]=x\} \text {. }
$$

It is clear that this adjustment ensures that (2) is invariantly true.

The information in $M$ is not sufficient to perform the adjustment; it is necessary to associate with each member $m$ the set $I(m)=\{i \mid m[i]=m\}$. Given this information, the primality test and its dependent actions can be written abstractly as

$$
\begin{aligned}
& \text { if } I(x) \neq \varnothing\{\text { i.e. } x \in M\} \\
& \text { then } \forall i \in I(x) \text { do } I(x+p[i]):=I(x+p[i]) \cup\{i\} \\
& \text { else begin } \mathrm{k}:=\mathrm{k}+\mathrm{l} \text {; } \\
& \text { end } \\
& \mathrm{p}[\mathrm{k}]:=\mathbf{x}
\end{aligned}
$$

This algorithm ensures that the relation

$$
I(x)=\left\{i \mid x=\text { the least multiple of } p_{i} \geq x\right\}
$$

holds invariantly before the test $x \in M$ (provided $x$ is incremented by 1 ; alternatively, see the second remark in \$5). 
We now look fox a suitable implementation of the above algorithm. We write $I(y)$ for the number of primes not greater than $y$, and $y$ ] for the integer part of $y$. Since from (2)

$$
M \in\{m \mid x \leq m<x+p[1 i m-1]\} \text {, }
$$

and $\lim -1 \leq \bar{\Pi}(\sqrt{N})$, a circular array $c[0 .[\sqrt{N}]]$ seems appropriate, since $|\mathrm{M}| \leq \mathrm{P}_{\pi}(\sqrt{\mathrm{N}}) \leq\lfloor\sqrt{\mathrm{N}}]$

Also, the elements of this array can be (pointers to) lists representing the sets $I(m)$. An index xp defines the current position of $x$, so that

$$
I(m) \text { is-represented-by } C[(x p+m-x) \text { mod }(I+[\sqrt{N}))]
$$

is the appropriate invariant for the data structure. Figure 2 is a typical snapshot of this data structure. It is now easy to implement the abstract operations used above.

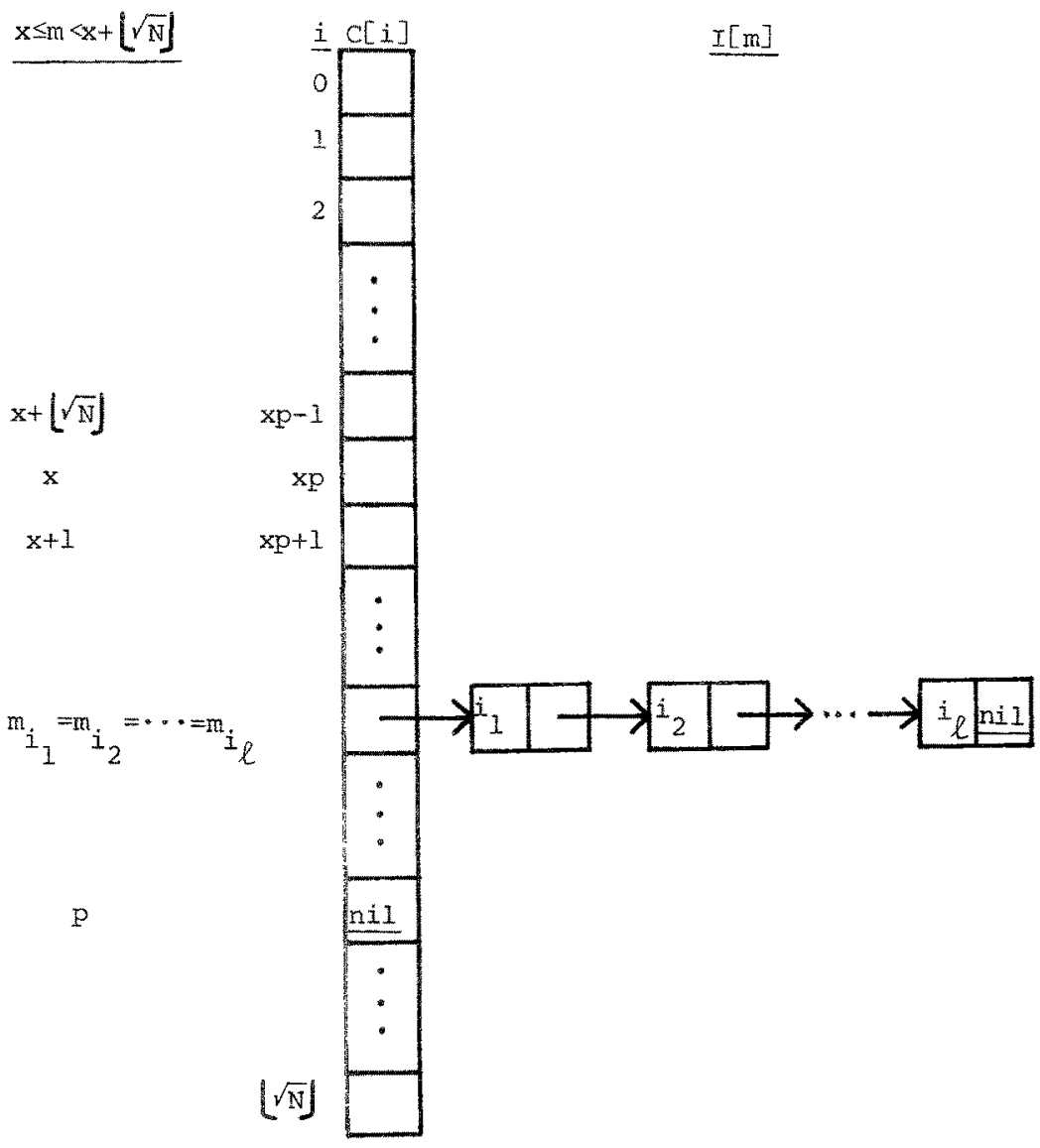

FIGURE 2: A SNAPSHOT OF THE DATA STRUCTURE FOR THE SET OF MULTTPLES 
At this point the programmer would likely want to obtain a feeling for the (absolute and relative) space and time efficiencies of the two algorithms. We consider such analyses to be an important part of the programmer's task. They are essential for comparing alternative algorithms, and as a check on intuitions. The latter use is well illustrated by McMaster (1978), whose analyses show that a series of refinements performed by Dijkstra to speed up a certain algorithm have almost no effect. Also, absolute analyses of complexity can be a useful guide to the "distance" of an algorithm from the best possible; $1 . e$. as to whether it is worthwhile seeking a more efficient algorithm.

Given the derivation of the current algorithm, a relative analysis with respect to program 1 is not difficult, and indicates an increase in time-efficiency. The operations used to mark composites (i.e. form the sets $I(m)$ ) in the transformed program are done implicitly in the original because m[i] steps through the multiples of $p_{i}$. But extra operations are incurred when $\mathrm{m}[\mathrm{i}]$ is tested without being incremented. The results referred to in the next section substantiate this informal argument. However it is apparent that space is wasted with nil pointers, because at most $\Pi(\sqrt{\mathrm{x}}) \leq \Pi(\sqrt{\mathrm{N}})=0(\sqrt{\mathrm{N}} / \operatorname{logN})$ primes are "active". So it is worth investigating whether the second algorithm can be transformed to increase its storage efficiency (it needs $0(\sqrt{\mathrm{N}} \log \mathrm{N})$ bits at present).

A fertile source of program transformations is the procedure of abstracting away from inessential time-ordering of operations (this is a strong motivating factor in the recent work of Dijkstra (1976)). In the algorithm under consideration a case in point is the process of adding $i$ to $I(x+p[i])$ for all $i \in I(x)$ before considering further value(s) of $x$. It seems a good idea to avoid numerous list operations by marking $x+n \cdot p[i]$ as composite for $n=1,2, \ldots$, and only adding $i$ to $I(x+n \cdot p[i])$ when $x+(n+1) \cdot p[i]$ falls outside $C$. This process can then be repeated for the remaining values $i \in I(x)$. When all members of $I(x)$ have been processed, successive values of $x$ can be examined and accepted as primes if unmarked, until the first value with $I(x)$ non-empty is found, and/or lim needs to be increased to "activate" a new potential factor. The processing of all $i \in I(x)$ is then repeated.

Given this new time-ordering of operations, it is not difficult to see that the data structure of Figure 2 is no longer appropriate, because only elements near the bottom of the (virtual) array are used for (pointing to) lists of indices of active primes. Furthermore, if the circularity of the array is removed, so that numbers $\mathrm{x}$ are processed in successive intervals of length $[\sqrt{\mathrm{N}}]$, the first multiple of $\mathrm{p}$ [i] outside the current interval can be stored in an array as $\mathrm{m}[i]$. Then, when the next interval is to be processed, the marking of all composites $m[i]+n \cdot p[i]$ can be done in any order of the indices $i$. Now each element of the array $C$ need only be a Boolean (one bit) which records whether or not the corresponding value was marked as a composite. The program we have arrived at is given in Figure 3. 


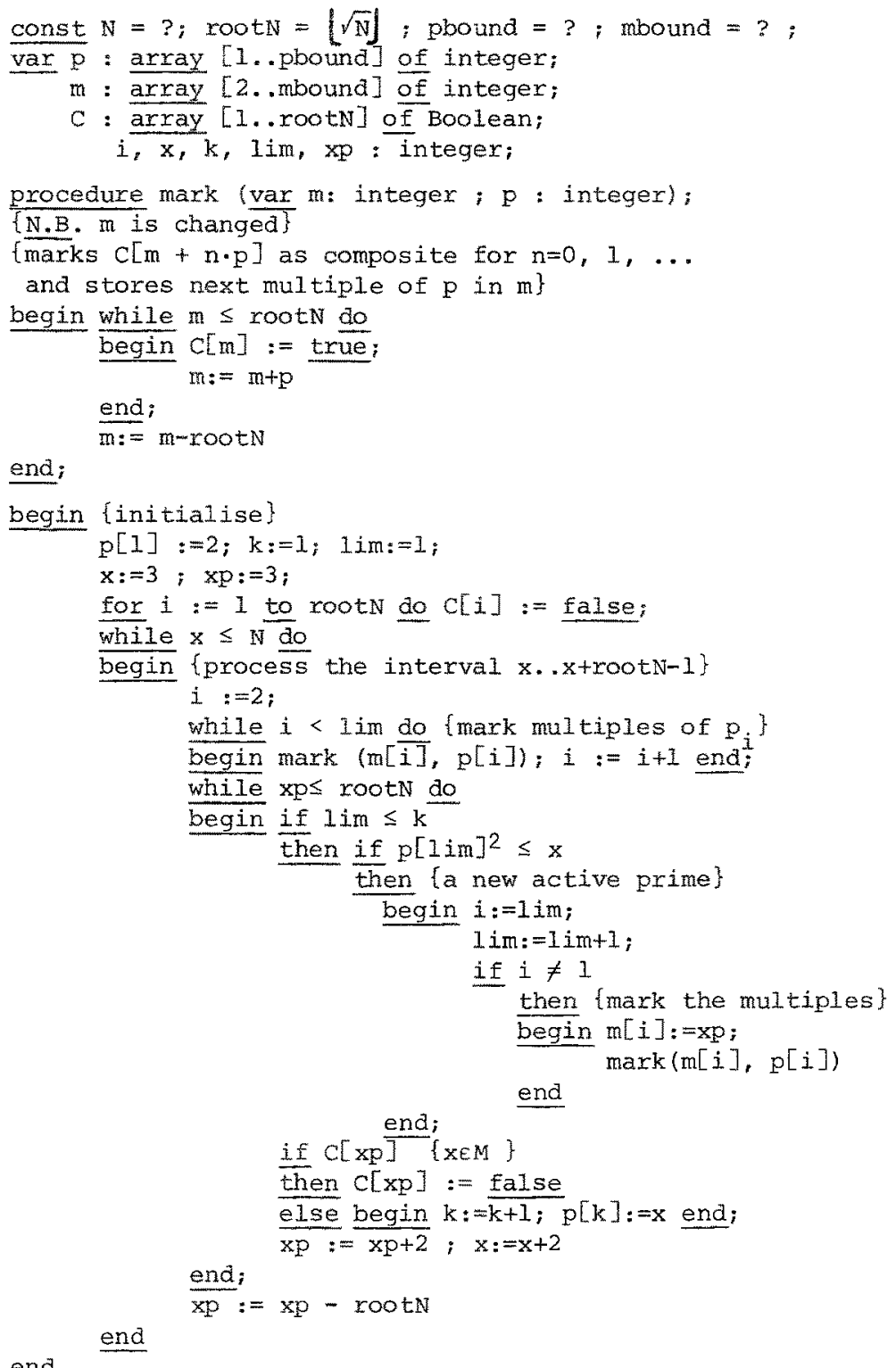

\section{FIGURE $3:$ PROGRAM 2}

The bounds on the arrays $\mathrm{p}$ and $\mathrm{m}$ can be determined when the time- and space-complexities of programs 1 and 2 are calculated. Further transformations can be done to obtain local increases in efficiency in program 2, which is essentially the "segmented sieve of Eratosthenes" of Bays and Hudson (1977). This seems to be the best practical 
algorithm for very large values of $N$, although we have given

algorithms with lower bit complexities in Pritchard (1979b, 1979c).

\section{COMPLEXITY ANALYSES}

In order not to burden the reader with undue mathematical detail, we simply state the results of our complexity analyses in the time domain. These analyses can be found in Pritchard (1979a). The space requirements of the two programs are much easier to calculate, and we proceed to do so. It is only necessary to know that $\Pi(y) \sim y / \log y$.

Program 1 takes $0\left(\sqrt[N]{N} / \log ^{3} N\right)$ additions, and this estimate is tight. As for storage, it requires $\pi(\sqrt{N})$ multiples and $\pi(\sqrt{N})$ primes (the rest need be printed only). The multiples can each be stored in $\log _{2} \mathrm{~N}$ bits, and the primes each take $\frac{1}{2} \log _{2} \mathrm{~N}$ bits since they are not greater than $\sqrt{\mathrm{N}}$. So the storage requirement is $\sim 3 \log _{2}$ e $\sqrt{\mathrm{N}}$ bits.

Program 2 requires $O(N \log \log N)$ additions, which is much better than the $0\left(\mathrm{~N} \sqrt{\mathrm{N}} / \log ^{3} \mathrm{~N}\right)$ additions required by program 1 . What is its storage requirement? Well, 3 arrays are used: $p, m$ and $c$. pbound $=\pi(\mathrm{N})$, but only the first $\pi(\sqrt{\mathrm{N}})$ primes need to be kept, requiring $\pi(\sqrt{\mathrm{N}}) \cdot \log _{2} \sqrt{\mathrm{N}}$ bits. mbound is also $\pi(\sqrt{\mathrm{N}})$, so another $\pi(\sqrt{\mathrm{N}}) \cdot \log _{2} \sqrt{\mathrm{N}}$ bits are needed for $\mathrm{m}$. Lastly, c takes $[\sqrt{\mathrm{N}}]$ bits. Hence the total storage requirement is $\sim\left(2 \log _{2} e+1\right) \sqrt{\mathrm{N}}$ bits, which is slightly smaller than that of program 1 . These figures unequivocally show the superior efficiency of the alternative program.

\section{FINAL REMARKS}

The importance of refinement in the development of clear and correct programs is indubitable. This recognition is due in large part to the efforts of Dijkstra and wirth. Our point in re-examining their primes problem is to show the importance of program transformations in the development of efficient programs; they add an extra (lateral) dimension to the programing process.

Dijkstra (1972), in a postscript to his development of program I, notes that $\mathrm{m}[\mathrm{i}]$ can in fact be increased by $2 \cdot \mathrm{p}[\mathrm{i}]$ because only odd values of $x$ are tested, and hence only odd multiples of $p_{i}$ are needed. This move carries over directly to the transformed algorithm - i is added to the set $I(x+2 \cdot p[i])$ instead of $I(x+p[i])$. No increase in storage results because $c$ need only contain odd values in the interval $x \leq m<x+2 \cdot p[1 i m-1]$, and there are at most $[\sqrt{\mathrm{N}}]$ of these.

Finally, we note that an attempt is made to formally capture and certify the original (and fundamental) transformation of program 1 in Pritchard (1978), where two other examples of this high-level transformation are also examined. 


\section{REFERENCES}

BAYS, C., and HUDSON, R.H. (1977): "The segmented sieve of Eratosthenes and Primes in Arithmetic Progression to $10^{12 *}$. B.I.T., Vol. 17, pp 121-127.

DIJKSTRA, E.W. (1972): "Notes on Structured Programing".

In "Structured Programming", Academic Press, New York, pp. 1-82.

DIJKSTRA, E.W. (1976): "A Discipline of Programming". Prentice-Ha11, Englewood Cliffs, New Jersey.

HARDY, G.H., and WRIGHT, E.M. (1965): "An Introduction to the Theory of Numbers" "Fourth edition, O.U.P." London.

HOARE, C.A.R. (1972): "Notes on Data Structuring". In "Structured Programming", Academic Press, New York, pp.83-174.

MCMASTER, C.I. (1978): "An Analysis of Algorithms for the Dutch National Flag problem". Comm. ACM, Vol. 21, No. 10, pp. 842-846.

PRITCHARD, F.A. (1978): "Program Design Via Multiple-Pass Algorithms". Typescript, Canberra, dated May 1978.

PRTTCHARD, P.A. (1979a): "On the Prime Example of Programming". In "Variations on a scheme of Eratosthenes", Tech. Report No. 8, Dept. of Computer Science, Univ. of queensland, pp. 1-2l.

PRITCHARD, P.A. (1979b): "On a Proposed Method of Generating Prime Numbers". In "Variations on a Scheme of Eratosthenes", Tech. Report No. 8, Dept. of Computer Science, Univ. of Queensland, pp. 22-28.

PRITCHARD, P.A. (1979c): "A Fast Linear Sieve for Finding prime Numbers". In "Variations on a Scheme of Eratosthenes", Tech. Report. No. 8, Dept. of Computer Science, Univ. of queensland, pp. $29-41$.

WIRTH, N. (1973): Systematic Programming: An Introduction. Prentice-Hall, Englewood Cliffs, New Jersey. 